\title{
Periodic Limb Movements during Sleep: Review of Physiology and Pathology
}

\author{
Brian Koo \\ Department of Neurology, Case Western Reserve University School of Medicine, Cleveland, OH, USA
}

Received November 30, 2012 Revised December 14, 2012

Accepted December 14, 2012

Address for correspondence

Brian Koo, MD

Department of Neurology,

University Hospitals

Case Medical Center,

11100 Euclid Avenue,

Cleveland, OH 44106, USA

Tel: +1-216-844-3422

Fax: +1-216-983-0792

E-mail: koobri@gmail.com
Periodic limb movements during sleep (PLMS) are spontaneously occurring movements of the legs which occur repetitively throughout the sleeping period. The clinical significance of these movements is unclear but when they occur frequently, sleep fragmentation can result. PLMS occur commonly in persons with the restless legs syndrome and also in the elderly even without sleep complaints. PLMS are not specific and can occur in the setting of several different diseases, including essential hypertension, stroke and sleep apnea. In this review, we cover briefly the epidemiology of PLMS and then go more into depth concerning physiology and then adverse effects associated with PLMS.

J Korean Sleep Res Soc 2012;9:23-27

\section{Introduction}

Periodic limb movements during sleep (PLMS) are spontaneously occurring movements of the legs which occur repetitively throughout the sleeping period. Individual limb movements last up to five seconds and consist of foot dorsiflexion and less often flexion at the knee or hip. ${ }^{1}$ Movements occur approximately every thirty seconds and in severe cases can number well into the hundreds each night. ${ }^{2}$ PLMS were first described in 1953 by Symonds, who referred to the movements as 'nocturnal myoclonus' and were first recorded electrographically by Lugaresi in what is now part of standard polysomnography (PSG). ${ }^{3,4}$ PLMS are common even in the absence of sleep complaints, especially in the elderly and are often discovered coincidentally on PSG. ${ }^{5}$ The movements can co-occur with a myriad of disorders including sleep apnea, stroke and even hypertension but occur most prevalently in the restless legs syndrome (RLS) in which PLMS are present in $80-90 \%$ of cases. ${ }^{6-9}$ The purpose of the following publication is to present a general overview of PLMS, reviewing its epidemiology, pathophysiology, genetics and clinical pertinence.

\section{Definitions}

It is worth clarifying that PLMS is the acronym for PLMS while PLMs refer to individual periodic limb movements. Also note that the 'L' in PLMS stands for limb not leg although the leg extremity is more commonly involved than the arm. PLMS differs from PLMD or periodic limb movement disorder which refers to the presence of PLMS plus daytime symptoms which may include sleepiness or fatigue. The entity of PLMD will not be reviewed here. Although the reader is referred to other sources for a review of PLMD, he/ she is reminded of the controversy as to whether PLMD really exists. ${ }^{10,11}$

Periodic limb movements during sleep is an electrographic phenomenon and can be reliably verified only with the use of polysomnography or other device specifically designed to detect limb movements over time. Coleman first presented criteria for the scoring of PLMS in 1982 which was later revised by the American Sleep Disorders Association in 1993 and then again by the World Association of Sleep Medicine (WASM) in 2006. ${ }^{12-14}$ To be considered a periodic limb movement, individual events had to last between 0.5 and 5 seconds, be within 5 to 90 seconds of each other and occur in a cluster of 4 or more. In 2006, the International Restless Legs Syndrome Study Group and WASM recommended to add 
an amplitude criterion in that individual movements should be scored only when there was an $8 \mu \mathrm{V}$ increase from baseline. Also the maximum duration was extended to 10 seconds. Additionally, rules were clarified for the scoring of individual movements with arousal and in the proximity of disordered breathing events. ${ }^{14}$

The frequency of PLMS are often presented in a periodic limb movement index (PLMI) as the number of PLMs per hour of sleep. Arousals are scored when they overlap before or after the event within 0.5 seconds. The number of PLMs occurring with arousal is reported in a periodic limb movement arousal index (PLMAI) as the number of PLMs occurring with arousal per hour of sleep.

\section{Epidemiology}

The prevalence of PLMS is likely between $5 \%$ and $11 \%$ of the general population. ${ }^{15,16}$ In a sample of randomly chosen adults between the ages of 18 and 65 years, 7.6\% of 592 individuals had PLMS recorded by PSG. Interestingly, PLMS was significantly more common in Caucasians than African Americans $(9.3 \%$ vs. $4.3 \%) .{ }^{17}$ The prevalence of PLMS seems to increase in the elderly, as $45 \%$ of 427 elderly over 65 years were found to have PLMS on PSG; a similar number of persons with PLMS was found in a community dwelling elderly female population. $^{18,19}$

Periodic limb movements during sleep most commonly co-occurs with RLS; in a group of 133 subjects with RLS, PLMI greater than 5 was seen in $80 \%$ and $88 \%$ of subjects on consecutive PSG nights. ${ }^{20}$ PLMS frequency increases with advancing age in RLS after the age of 15 and then again after age $65{ }^{21}$ There is conflicting data when considering the correlation between PLMS frequency and severity of RLS. ${ }^{22,23}$ PLMS also can be seen in other sleep disorders including obstructive sleep apnea, narcolepsy and REM sleep behavior disorder or in disorders not affecting sleep including essential hypertension, congestive heart failure and end-stage renal disease. ${ }^{8,24-28}$ PLMS seem to arise or become more frequent with the use of many antidepressant medications, including tricyclic antidepressant, fluoxetine and venlafaxine but not buproprion. ${ }^{29-33}$

\section{Pathophysiology}

Periodic limb movements during sleep most often occur in the legs and most often consist of ankle and great toe dorsiflexion with movement at the knee and hip being less frequent. In total, the movement resembles triple flexion seen with upper motor neuron lesioning. The muscle most commonly involved is the tibialis anterior followed by the extensor digitorum brevis and biceps femoris. ${ }^{34}$ The sequence of muscle activation is variable and can consist of different permutations of the above muscles. ${ }^{35}$ Early electromyographic studies suggest that the recruitment pattern may represent either rostral or causal spinal propagation between L3 and S1. ${ }^{36}$ Additional evidence for a spinal localization for PLMS is increased spinal cord excitability as indicated by lower threshold for the spinal flexor reflex in subjects with RLS and PLMS compared to normal controls. ${ }^{37}$

Physiologically, PLMS seem to be related to several different systems including cerebral, cardiovascular and autonomic. Individual limb movements of a PLMS cluster are predictably preceded by increased delta frequency cerebral activity and then followed by faster electroencephalographic frequencies. ${ }^{38}$ As this sequence recurs a cyclic alternating pattern, reflecting arousal rhythms can be seen in NREM sleep. ${ }^{39} \mathrm{~A}$ parallel process co-occurs in the autonomic and cardiovascular systems. Heart rate begins to accelerate 2-3 cardiac cycles before a PLM, peaks 4-5 cycles following a PLM and falls below pre-movements rates 8-10 cycles after a PLM. ${ }^{40}$ Consistent with this finding, heart rate variability measures in the setting of PLMS demonstrate both vagal and sympathetic activation but with predominance of sympathetic activity. ${ }^{41,42}$ Blood pressure also increases discretely with each individual limb movement on the order of 10 to $20 \mathrm{~mm} \mathrm{Hg}$ systolic units, more toward the upper limit when associated with arousal. ${ }^{43,44}$

Consistent findings of PLMS associated autonomic activation have spurred an increased focus on the potential long term cardiovascular risk conferred by PLMS. Studies of the two largest experimental sleep cohorts, Sleep Heart Health and Wisconsin cohorts, have shown an association between self-reported RLS and prevalent cardiovascular disease, including coronary heart disease and stroke. ${ }^{45,46}$ In neither of these studies was PLMS measured. In a study of community dwelling older men followed for more than 4 years, PLMS (measured with PSG), especially when associated with arousal, was associated with an approximately $26 \%$ increased risk of incident cardiovascular disease which included myocardial infarction, coronary artery disease, stroke and peripheral vascular disease. In this study of elderly men, there was no information regarding self-report RLS symptoms. ${ }^{47}$ The optimal study to look at whether PSG measured PLMS and selfreported RLS is associated with cardiovascular disease has not been carried out.

For the above study of PLMS and incident cardiovascular disease, the question remains as to whether PLMS is simply a marker for poor cardiovascular health, especially in this study since it was done in elderly men. Furthermore, PLMS associated with RLS may have differing significance than PLMS occurring in the absence of RLS. As mentioned above, PLMS can be associated with a myriad of different disorders even in 
the absence of RLS. It is important for the scientific community to determine if PLMS in these different circumstances have distinct physiologic etiologies with distinct pathologic consequences.

\section{Genetics}

The genetics of RLS is complex and incompletely understood. Early studies employed genetic linkage techniques in large families afflicted with RLS to identify more than eight different loci. ${ }^{48}$ More recently genome-wide association technology has been employed in a larger number of individuals carefully phenotyped for RLS. These studies identified variants within several different candidate genes, MEIS1, BTBD9, MAP2K5, LBXCOR1 and PTPRD; however, it still remains unclear what role these genes play in RLS. ${ }^{49-54}$ Only one of these studies employed the measurement of PLMS in addition to RLS symptomatology in the phenotyping of RLS individuals. ${ }^{53}$ This study assessed populations in Atlanta, Georgia and in Iceland and revealed two variants in the BTBD9 gene that were significantly associated with RLS/PLMS. Still, it is unclear how these genes confer risk of developing PLMS.

\section{Effects on Sleep}

Periodic limb movements during sleep have a clear and reproducible distribution across the night in relation to sleep stages. Especially in the RLS but even in some subjects without RLS, periodic movements of the legs often begin in quiet wakefulness before sleep has begun. ${ }^{2}$ The number of movements during quiet wakefulness per unit time often exceeds what is seen in any other sleep stage, so careful review of wake PSG epochs is important in patients with PLMS. PLMS are most frequent and individual movements are longest during light NREM sleep and become less frequent and shorter with progression to NREM stage 2 and then NREM stage 3. PLMS are least frequent and shortest during REM sleep. The frequency of PLM-arousal complexes follows this same distribution being most frequent in light NREM stage 1 sleep and least frequent in slow wave and REM sleep. ${ }^{55}$

It seems logical that movements of the upper and lower legs can cause arousal but such a progression may not be the case. Although cortical arousal and PLMS do seem to be related temporally, causality has not been demonstrated either way. Spectral and time series analysis of PLMS, EEG and EKG demonstrate that EEG delta and alpha band activity as well as heart rate increases preceding electromyographic potentials exhibited by PLMS. ${ }^{42}$ In other words, the occurrence of PLMS is heralded by changes in EEG and EKG power spectrums. Further evidence that EEG arousal and PLMS are as- sociated but not necessarily causal comes from a study that shows PLMS and EEG arousal can be pharmacologically isolated. More specifically, in subjects with RLS on successive nights, treatment with a dopamine agonist decreases the frequency of PLMS but not arousal; conversely, treatment with clonazepam decreases the frequency of arousal but not PLMS. ${ }^{56}$

Sleep does seem to be affected by PLMS. Many studies which investigate the effect of PLMS on sleep were carried out in subjects with RLS, thus it is not known to what extent the PLMS rather than RLS disrupts sleep. Nevertheless, some of these studies are worth mentioning in this monograph. RLS patients compared to normal controls display a prolonged sleep latency, decreased total sleep time and efficiency while having increased wake after sleep onset. ${ }^{57}$ Pharmacologic treatment of the RLS results in an increase on total sleep time and efficiency, a decrease in sleep latency and wake after sleep onset as well as a significant reduction in the frequency of PLMS. ${ }^{58}$ Also in these studies there was an improvement in subjective sleep quality and to a lesser extent quality of life.

Clearly, when there is PLMS in association with RLS and sleep disruption, there is evidence that pharmacologic treatment can be beneficial. The situation is not as clear when there is PLMS in the absence of RLS. Small open label studies suggest that treatment of PLMS in the presence of sleep disturbance is often successful in reducing limb movements but less consistently is sleep efficiency or arousal improved. ${ }^{57,59,60}$ In the 2012 Associated Professional Sleep Societies conference, there was a symposium based upon the question of whether or not to treat PLMS. The expert consensus was that there is no evidence that treating PLMS in the absence of RLS is beneficial but that in individual cases, treatment could be considered if there is sleep disruption and/or hypersomnolence associated with PLMS.

\section{Conclusions}

Periodic limb movements during sleep are a curious series of movements that occur in sleep. The periodicity displayed in these leg movements also seems to occur in other physiologic systems which include cerebral, cardiac and respiratory organs. In sleep, this periodicity is pervasive and seems to underscore an importance to this measure, but to what extent PLMS is significant is unclear. PLMS themselves seem to track with cardiovascular disease but whether they are a cause of such dysfunction or just a marker of poor cardiovascular health is also not clear. As these basic mechanisms of PLMS are unclear so too are the immediate clinical implications of these movements. There is no clear indication for the treatment of PLMS but medical treatment of these movements could be taken on a case by case basis. Genetic, epide- 
miologic and physiologic research in this interesting area will help to clarify some of these important questions in the years to come.

\section{REFERENCES}

1. Wechsler LR, Stakes JW, Shahani BT, Busis NA. Periodic leg movements of sleep (nocturnal myoclonus): an electrophysiological study. Ann Neurol 1986;19:168-173.

2. Pollmächer T, Schulz H. Periodic leg movements (PLM): their relationship to sleep stages. Sleep 1993;16:572-577.

3. Symonds CP. Nocturnal myoclonus. J Neurol Neurosurg Psychiatry 1953;16:166-171.

4. Lugaresi E, Coccagna G, Mantovani M, Lebrun R. Some periodic phenomena arising during drowsiness and sleep in man. Electroencephalogr Clin Neurophysiol 1972;32:701-705.

5. Bliwise D, Petta D, Seidel W, Dement W. Periodic leg movements during sleep in the elderly. Arch Gerontol Geriatr 1985;4:273-281.

6. Warnes H, Dinner DS, Kotagal P, Burgess RC. Periodic limb movements and sleep apnoea. J Sleep Res 1993;2:38-44.

7. Kang SY, Sohn YH, Lee IK, Kim JS. Unilateral periodic limb movement in sleep after supratentorial cerebral infarction. Parkinsonism Relat Disord 2004;10:429-431.

8. Espinar-Sierra J, Vela-Bueno A, Luque-Otero M. Periodic leg movements in sleep in essential hypertension. Psychiatry Clin Neurosci 1997;51:103-107.

9. Abetz L, Allen R, Follet A, et al. Evaluating the quality of life of patients with restless legs syndrome. Clin Ther 2004;26:925-935.

10. Hornyak M, Feige B, Riemann D, Voderholzer U. Periodic leg movements in sleep and periodic limb movement disorder: prevalence, clinical significance and treatment. Sleep Med Rev 2006;10:169-177.

11. American Academy of Sleep Medicine. International classification of sleep disorders, revised: Diagnostic and coding manual. Chicago, IL: American Academy of Sleep Medicine, 2001.

12. Coleman RM. Periodic movements in sleep (nocturnal myoclonus) and restless legs syndrome. In: Guilleminault C. Sleeping and waking disorders: indications and techniques. Menlo Park: AddisonWesley, 1982;265-295.

13. Recording and scoring leg movements. The Atlas Task Force. Sleep 1993;16:748-759.

14. Zucconi M, Ferri R, Allen R, et al. The official World Association of Sleep Medicine (WASM) standards for recording and scoring periodic leg movements in sleep (PLMS) and wakefulness (PLMW) developed in collaboration with a task force from the International Restless Legs Syndrome Study Group (IRLSSG). Sleep Med 2006;7:175-183.

15. Kales A, Wilson T, Kales JD, et al. Measurements of all-night sleep in normal elderly persons: effects of aging. J Am Geriatr Soc 1967;15:405414.

16. Bixler EO, Kales A, Vela-Bueno A, Jacoby JA, Scarone S, Soldatos CR. Nocturnal myoclonus and nocturnal myoclonic activity in the normal population. Res Commun Chem Pathol Pharmacol 1982;36:129-140.

17. Scofield H, Roth T, Drake C. Periodic limb movements during sleep: population prevalence, clinical correlates, and racial differences. Sleep 2008;31:1221-1227.

18. Ancoli-Israel S, Kripke DF, Klauber MR, Mason WJ, Fell R, Kaplan O. Periodic limb movements in sleep in community-dwelling elderly. Sleep 1991;14:496-500.

19. Claman DM, Redline S, Blackwell T, et al. Prevalence and correlates of periodic limb movements in older women. J Clin Sleep Med 2006;2: 438-445.

20. Montplaisir J, Boucher S, Poirier G, Lavigne G, Lapierre O, Lespérance P. Clinical, polysomnographic, and genetic characteristics of restless legs syndrome: a study of 133 patients diagnosed with new standard criteria. Mov Disord 1997;12:61-65.
21. Ferri R, Manconi M, Lanuzza B, et al. Age-related changes in periodic leg movements during sleep in patients with restless legs syndrome. Sleep Med 2008;9:790-798.

22. Garcia-Borreguero D, Larrosa O, de la Llave Y, Granizo JJ, Allen R. Correlation between rating scales and sleep laboratory measurements in restless legs syndrome. Sleep Med 2004;5:561-565.

23. Hornyak M, Kopasz M, Voderholzer U, et al. Relationship of the restless legs syndrome rating scale scores to objective and subjective measures of sleep. Sleep 2004;27:A309.

24. Baker TL, Guilleminault C, Nino-Murcia G, Dement WC. Comparative polysomnographic study of narcolepsy and idiopathic central nervous system hypersomnia. Sleep 1986;9 (1 Pt 2):232-242.

25. Baran AS, Richert AC, Douglass AB, May W, Ansarin K. Change in periodic limb movement index during treatment of obstructive sleep apnea with continuous positive airway pressure. Sleep 2003;26:717-720.

26. Fantini ML, Michaud M, Gosselin N, Lavigne G, Montplaisir J. Periodic leg movements in REM sleep behavior disorder and related autonomic and EEG activation. Neurology 2002;59:1889-1894.

27. Hanly PJ, Zuberi-Khokhar N. Periodic limb movements during sleep in patients with congestive heart failure. Chest 1996;109:1497-1502.

28. Benz RL, Pressman MR, Hovick ET, Peterson DD. Potential novel predictors of mortality in end-stage renal disease patients with sleep disorders. Am J Kidney Dis 2000;35:1052-1060.

29. Myers BA, Klerman GL, Hartmann E. Nocturnal cataclysms with myoclonus: a new side effect of clomipramine. Am J Psychiatry 1986;143: 1490-1491.

30. Garvey MJ, Tollefson GD. Occurrence of myoclonus in patients treated with cyclic antidepressants. Arch Gen Psychiatry 1987;44:269-272.

31. Dorsey CM, Lukas SE, Cunningham SL. Fluoxetine-induced sleep disturbance in depressed patients. Neuropsychopharmacology 1996;14: 437-442.

32. Salín-Pascual RJ, Galicia-Polo L, Drucker-Colín R. Sleep changes after 4 consecutive days of venlafaxine administration in normal volunteers. J Clin Psychiatry 1997;58:348-350.

33. Yang C, White DP, Winkelman JW. Antidepressants and periodic leg movements of sleep. Biol Psychiatry 2005;58:510-514.

34. Trenkwalder C, Bucher SF, Oertel WH. Electrophysiological pattern of involuntary limb movements in the restless legs syndrome. Muscle Nerve 1996;19:155-162.

35. de Weerd AW, Rijsman RM, Brinkley A. Activity patterns of leg muscles in periodic limb movement disorder. J Neurol Neurosurg Psychiatry 2004;75:317-319.

36. Plazzi G, Vetrugno R, Meletti S, Provini F. Motor pattern of periodic limb movements in sleep in idiopathic RLS patients. Sleep Med 2002;3 Suppl:S31-S34.

37. Bara-Jimenez W, Aksu M, Graham B, Sato S, Hallett M. Periodic limb movements in sleep: state-dependent excitability of the spinal flexor reflex. Neurology 2000;54:1609-1616.

38. Ferrillo F, Beelke M, Canovaro P, et al. Changes in cerebral and autonomic activity heralding periodic limb movements in sleep. Sleep Med 2004;5:407-412.

39. Parrino L, Boselli M, Buccino GP, Spaggiari MC, Di Giovanni G, Terzano MG. The cyclic alternating pattern plays a gate-control on periodic limb movements during non-rapid eye movement sleep. J Clin Neurophysiol 1996;13:314-323.

40. Winkelman JW. The evoked heart rate response to periodic leg movements of sleep. Sleep 1999;22:575-580.

41. Guggisberg AG, Hess CW, Mathis J. The significance of the sympathetic nervous system in the pathophysiology of periodic leg movements in sleep. Sleep 2007;30:755-766.

42. Sforza E, Pichot V, Barthelemy JC, Haba-Rubio J, Roche F. Cardiovascular variability during periodic leg movements: a spectral analysis approach. Clin Neurophysiol 2005;116:1096-1104.

43. Pennestri MH, Montplaisir J, Colombo R, Lavigne G, Lanfranchi PA. Nocturnal blood pressure changes in patients with restless legs syn- 
drome. Neurology 2007;68:1213-1218.

44. Siddiqui F, Strus J, Ming X, Lee IA, Chokroverty S, Walters AS. Rise of blood pressure with periodic limb movements in sleep and wakefulness. Clin Neurophysiol 2007;118:1923-1930.

45. Winkelman JW, Finn L, Young T. Prevalence and correlates of restless legs syndrome symptoms in the Wisconsin Sleep Cohort. Sleep Med 2006;7:545-552.

46. Winkelman JW, Shahar E, Sharief I, Gottlieb DJ. Association of restless legs syndrome and cardiovascular disease in the Sleep Heart Health Study. Neurology 2008;70:35-42.

47. Koo BB, Blackwell T, Ancoli-Israel S, et al. Association of incident cardiovascular disease with periodic limb movements during sleep in older men: outcomes of sleep disorders in older men (MrOS) study. Circulation 2011;124:1223-1231.

48. Trenkwalder C, Högl B, Winkelmann J. Recent advances in the diagnosis, genetics and treatment of restless legs syndrome. J Neurol 2009; 256:539-553.

49. Winkelmann J, Schormair B, Lichtner P, et al. Genome-wide association study of restless legs syndrome identifies common variants in three genomic regions. Nat Genet 2007;39:1000-1006.

50. Kemlink D, Polo O, Frauscher B, et al. Replication of restless legs syndrome loci in three European populations. J Med Genet 2009;46:315318.

51. Yang Q, Li L, Yang R, et al. Family-based and population-based association studies validate PTPRD as a risk factor for restless legs syndrome. Mov Disord 2011;26:516-519.
52. Schormair B, Kemlink D, Roeske D, et al. PTPRD (protein tyrosine phosphatase receptor type delta) is associated with restless legs syndrome. Nat Genet 2008;40:946-948.

53. Stefansson H, Rye DB, Hicks A, et al. A genetic risk factor for periodic limb movements in sleep. N Engl J Med 2007;357:639-647.

54. Winkelmann J, Czamara D, Schormair B, et al. Genome-wide association study identifies novel restless legs syndrome susceptibility loci on 2p14 and 16q12.1. PLoS Genet 2011;7:e1002171.

55. Karadeniz D, Ondze B, Besset A, Billiard M. Are periodic leg movements during sleep (PLMS) responsible for sleep disruption in insomnia patients? Eur J Neurol 2000;7:331-336.

56. Manconi M, Ferri R, Zucconi M, et al. Dissociation of periodic leg movements from arousals in restless legs syndrome. Ann Neurol 2012;71: 834-844.

57. Saletu M, Anderer P, Saletu B, et al. Sleep laboratory studies in periodic limb movement disorder (PLMD) patients as compared with normals and acute effects of ropinirole. Hum Psychopharmacol 2001;16:177-187.

58. Ferri R, Manconi M, Aricò D, et al. Acute dopamine-agonist treatment in restless legs syndrome: effects on sleep architecture and NREM sleep instability. Sleep 2010;33:793-800.

59. Grewal M, Hawa R, Shapiro C. Treatment of periodic limb movements in sleep with selegiline HCl. Mov Disord 2002;17:398-401.

60. Manconi M, Ferri R, Zucconi M, et al. Pramipexole versus ropinirole: polysomnographic acute effects in restless legs syndrome. Mov Disord 2011;26:892-895. 